\title{
Vaccine Wastage Assessment After Introduction of Open Vial Policy in Surat Municipal Corporation Area of India
}

\author{
Prakash B. Patel, Jayesh J. Rana*, Sunil G. Jangid, Neha R. Bavarva, Manan J. Patel, Raj Kumar Bansal
}

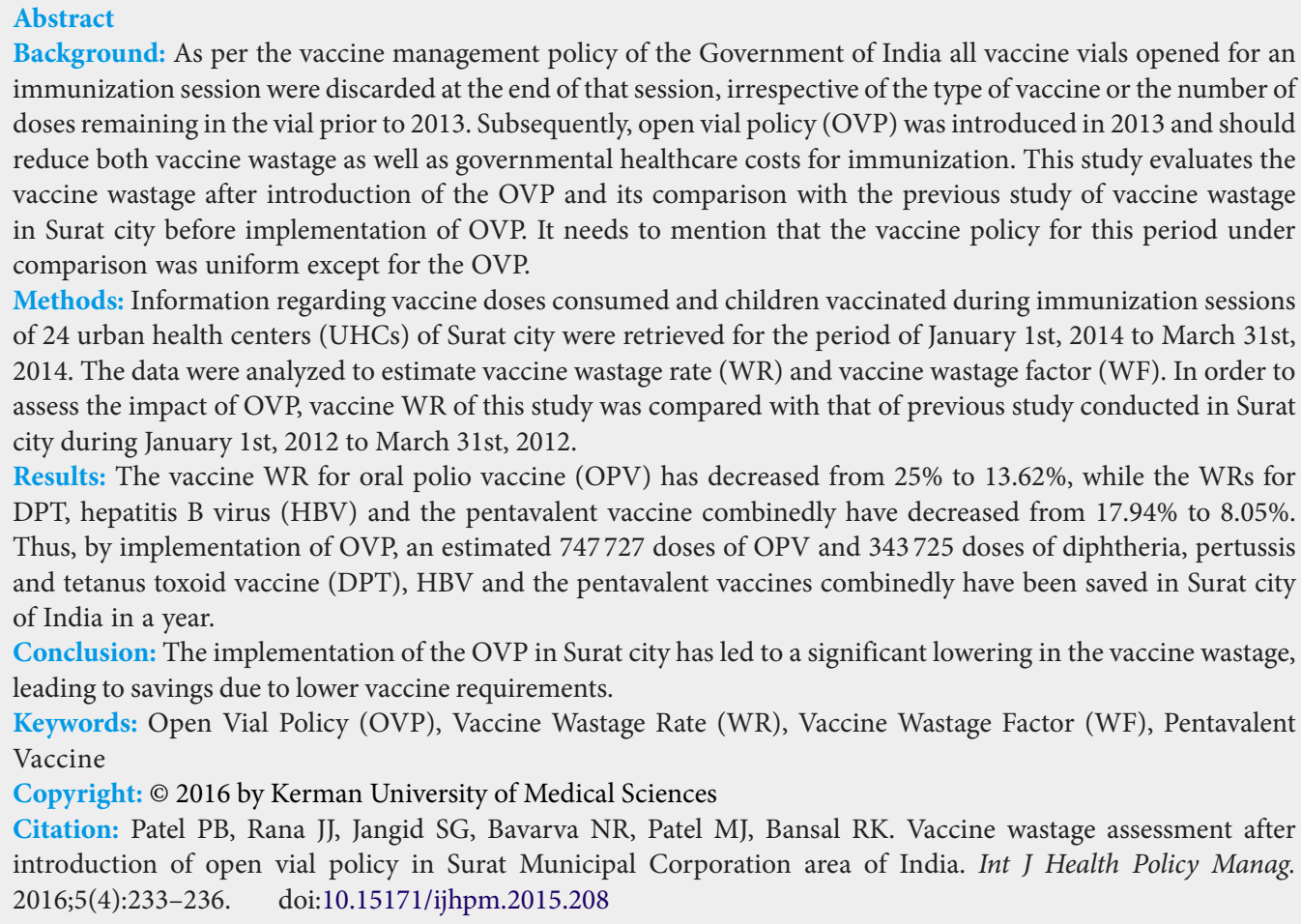

Background: As per the vaccine management policy of the Government of India all vaccine vials opened for an immunization session were discarded at the end of that session, irrespective of the type of vaccine or the number of doses remaining in the vial prior to 2013. Subsequently, open vial policy (OVP) was introduced in 2013 and should reduce both vaccine wastage as well as governmental healthcare costs for immunization. This study evaluates the vaccine wastage after introduction of the OVP and its comparison with the previous study of vaccine wastage in Surat city before implementation of OVP. It needs to mention that the vaccine policy for this period under comparison was uniform except for the OVP.

Methods: Information regarding vaccine doses consumed and children vaccinated during immunization sessions of 24 urban health centers (UHCs) of Surat city were retrieved for the period of January 1st, 2014 to March 31st, 2014. The data were analyzed to estimate vaccine wastage rate (WR) and vaccine wastage factor (WF). In order to assess the impact of OVP, vaccine WR of this study was compared with that of previous study conducted in Surat city during January 1st, 2012 to March 31st, 2012.

Results: The vaccine WR for oral polio vaccine (OPV) has decreased from 25\% to $13.62 \%$, while the WRs for DPT, hepatitis B virus (HBV) and the pentavalent vaccine combinedly have decreased from $17.94 \%$ to $8.05 \%$. Thus, by implementation of OVP, an estimated 747727 doses of OPV and 343725 doses of diphtheria, pertussis and tetanus toxoid vaccine (DPT), HBV and the pentavalent vaccines combinedly have been saved in Surat city of India in a year.

Conclusion: The implementation of the OVP in Surat city has led to a significant lowering in the vaccine wastage, leading to savings due to lower vaccine requirements.

Keywords: Open Vial Policy (OVP), Vaccine Wastage Rate (WR), Vaccine Wastage Factor (WF), Pentavalent Vaccine

Copyright: $\odot 2016$ by Kerman University of Medical Sciences

Citation: Patel PB, Rana JJ, Jangid SG, Bavarva NR, Patel MJ, Bansal RK. Vaccine wastage assessment after introduction of open vial policy in Surat Municipal Corporation area of India. Int J Health Policy Manag. 2016;5(4):233-236. doi:10.15171/ijhpm.2015.208

\section{Article History:}

Received: 30 April 2015

Accepted: 1 December 2015

ePublished: 8 December 2015

*Correspondence to:

Jayesh J. Rana

Email: dr.jayeshrana@gmail.com

\section{Key Messages}

Implications for policy makers

- Wastage rates (WRs) for oral polio vaccine (OPV) and injectable vaccines (DPT, hepatitis B [HBV] and pentavalent) have reduced by $50 \%$ after introducing open vial policy (OVP) in Surat city.

- We estimate that this reduction in vaccine wastage has led to an estimated saving of US\$0.7 million in the current health expenditure for immunization in Surat city.

- The vaccine WRs calculated in this study could prove useful for program managers in forecasting vaccine requirements elsewhere.

Implications for public

Vaccine supply for immunization is an essential expenditure to be borne by governments as health is an essential good for the development of a country. Financial constraints and unnecessary vaccine wastage in developing countries as India may translate into a numerically fewer children being vaccinated with the same resources. Therefore, it is prudent to minimize vaccine wastage and render additional doses to be made available for vaccination. This increases the cost-effectiveness of our immunization program, leads to an increased immunization coverage and increases the number of children protected from vaccine-preventable diseases. Thus, in this context open vial policy (OVP) would be helpful to decrease the economic costs and the burden of un-immunization and partial immunization of children in our society.

\section{Introduction}

Universal Immunization Program (UIP) was launched in 1985 in India with intent to immunize all eligible children by 1990. Our program guidelines dictated that a fresh vaccine vial was to be opened before the immunization session even if there was only one child requiring vaccination. ${ }^{1}$ The previous immunization policy had also stated that all vaccine vials opened for an immunization session had to be discarded at the end of that session, irrespective of the type of vaccine or the number of residual doses in the vial. ${ }^{2}$

Later as per the revised open vial policy (OVP), multi-dose vials of oral polio vaccine (OPV), Diphtheria, pertussis and tetanus toxoid vaccine (DPT), tetanus toxoid (TT), diphtheria and tetanus toxoid (DT), pentavalent, hepatitis B virus (HBV) and liquid formulations of Haemophilus influenzae type b (Hib) vaccines from which one or more doses have been 
removed during an immunization session could be used in successive sessions for a maximum of 4 weeks, provided that all of the World Health Organization (WHO) requirements for effectiveness and temperature stability were met. ${ }^{3}$

The WHO reports that over $50 \%$ of vaccine are wasted globally. ${ }^{4}$ Over-ordering of vaccines is the leading problem in the distribution chain, and causes the majority of wastage. ${ }^{5}$ Thus vaccine wastage is an important factor in forecasting vaccine requirements and while placing vaccine orders. India being a developing economy needs to reduce avoidable vaccine wastage and wasteful budgetary requirements. Therefore, the introduction of new vaccine management policy (OVP, 2013) seems a prudent economic choice given reduced vaccine wastage and increased cost-benefit ratio of immunization. This study was conducted to compare vaccine wastage before and after introducing the OVP by the Surat Municipal Corporation (SMC) for Surat city of India.

We are well-aware that our vaccine supply is limited owing to budgetary constraints. Therefore, any vaccine wastage translates into fewer vaccine doses actually available to children and also a higher cost of vaccination. ${ }^{6}$ Any minimization of this cost would be a welcome step in fiscal planning. It needs mention that the Government of Gujarat introduced the pentavalent vaccine in UIP in January 2013 and OVP for multi-dose vials was made available from April 2013 in Gujarat.?

This article attempts to calculate the vaccine WRs after introducing OVP in SMC area and the impact of OVP on vaccine wastage by comparing it with the previous study in Surat city before implementation of OVP.

\section{Methods}

The data from urban heath centers (UHCs) of SMC has been analyzed for the vaccine wastage for various vaccines. The information regarding vaccine doses consumed during immunization sessions and number of vaccinated children were retrieved from the immunization registers for the period of January 1st, 2014 to March 31st, 2014 (ie, after introduction of OVP) maintained by the public health nurses.

We have included 24 out of 40 UHCs, covering 1964 immunization sessions, of SMC as only these centers could provide complete immunization data. Six centers where daily in and out of vaccine vials as well as vaccination details of each session were not maintained properly were excluded and remaining ten centers had pleaded their temporary inability to provide a complete data due to their participation in ongoing public health programs.

The number of doses wasted for a particular vaccine was calculated by subtracting number of vaccinated children from the total vaccine doses consumed in a session. Here 'vaccine doses consumed in a session' imply the number of doses issued for the session and not returned back. We have included only the number of usable vials for our calculations. We were unable to find any unusable vaccine vials at various UHCs during our supervision visits as revealed by vaccine vial monitors. Further the vaccine vials discarded because lesser number of children available at vaccination session sites were in usable condition. Proportion of wasted doses from total consumed doses was considered as vaccine WR. ${ }^{2}$ Vaccine wastage factor (WF) was calculated by using the formula $[100 /(100 \text {-Vaccine WR })]^{2}$

The vaccine $\mathrm{WR}$ and vaccine WF of open multi-dose vials of all liquid vaccines were compared with that of previous study conducted by our department ie, Mehta et $\mathrm{al}^{8}$ in Surat city during January 1st, 2012 to March 31st, 2012 (ie, before introduction of OVP). In 2012 study, ${ }^{8}$ out of 36 health centers 24 had been included covering 2399 immunization sessions. During this study also 24 out of 40 centers were included covering 1964 immunization sessions. The method used for estimating WR and WF in both studies remain the same. As the pentavalent vaccine was not introduced at the time of previous study, the vaccine WR and vaccine WF for DPT, pentavalent, and HBV has been clubbed together in this study and compared with the combined vaccine WR and vaccine WF of DPT and HBV of the previous study. The pentavalent vaccine has replaced DPT and HBV and that is why they are deemed comparable and also all of these vaccines have similar administration techniques. However, the children who had received partial doses of DPT and HBV had continued with the past regimens.

\section{Results}

In 24 UHCs, total 1964 immunization sessions had been conducted during the study period. Six vaccines (bacille Calmette-Guerin vaccine [BCG], OPV, DPT, pentavalent, $\mathrm{HBV}$ and measles) were given to children. The information regarding the number of vaccine doses consumed for vaccination, children vaccinated, vaccine $W R$, and vaccine WF are depicted in Table 1. To measure the impact of OVP on vaccine wastage, only data regarding open multi-dose vials of liquid vaccines (OPV, DPT, pentavalent, and HBV) have been used and compared with the data of the previous study.

In the first quarter of 2014, 48501 doses of OPV were actually ingested by the children whereas 56150 doses of the vaccine were used. This translates into vaccine WR of $13.62 \%$ and vaccine WF of $1.16 \%$ for OPV. Earlier, in first quarter of 2012, vaccine WR and vaccine WF for OPV was $25 \%$ and $1.33 \%$, respectively. This shows a significant decline in vaccine WR

Table 1. Vaccine WR and Vaccine WF for Different Liquid Vaccines

\begin{tabular}{|c|c|c|c|c|c|}
\hline Vaccine (Reference Period) & Doses Consumed $^{\mathrm{a}}$ & $\begin{array}{c}\text { Actual Doses Received } \\
\text { by Children }\end{array}$ & Vaccine WR & Vaccine WF & $\begin{array}{c}P \text { Value (Using Z Test } \\
\text { of Proportion) }\end{array}$ \\
\hline OPV (January-March 2012) & 42290 & 31732 & $25.00 \%$ & 1.33 & \multirow{2}{*}{$<0.001$} \\
\hline OPV (January-March 2014) & 56150 & 48501 & $13.62 \%$ & 1.16 & \\
\hline DPT and HBV (January-March 2012) & 91249 & 74883 & $17.94 \%$ & 1.22 & \multirow{2}{*}{$<0.001$} \\
\hline DPT, HBV, and pentavalent (January-March 2014) & 51858 & 47686 & $08.05 \%$ & 1.09 & \\
\hline
\end{tabular}

Abbreviations: OPV, oral polio vaccine; HBV, hepatitis B virus; WR, wastage rate; WF, wastage factor; DPT, diphtheria, pertusis, and tetanus toxoid vaccine.

${ }^{a}$ These were the doses calculated by subtraction of vaccine doses issued before the vaccination session and doses received at the end of vaccination session.

${ }^{b}$ These were the doses actually received by the children based on the actual doses administered. These children have individual registration and addresses listed for each entry and are cross verifiable. 
and vaccine WF for OPV after implementation of OVP $(P<.001)$. It has been mentioned earlier that these vaccine doses wasted were usable as per the vaccine vial monitor. In first quarter of 2012, DPT and HBV vaccines were given separately. All children receiving first, second or third dose of DPT were provided with HBV vaccine while DPT booster was given without $\mathrm{HBV}(6,10$, and 14-week schedule followed for HBV). Combining both the vaccine, total 74883 doses were injected to children in the quarter and for that 91249 doses of vaccines were actually used.

In January 2013, pentavalent vaccine was introduced replacing both DPT and HBV in newly registered children. Those children who had already received one or more doses of DPT were continued on the same vaccine till second booster. In first quarter of 2014, 32092 doses of pentavalent, 14702 doses of DPT, and 892 doses of HBV were actually injected to the children. Thus, a total of 47686 injections were provided to the children and for which 51858 doses were used. This gives a vaccine WR of $8.05 \%$ and vaccine WF of $1.09 \%$.

Earlier, in first quarter of 2012, vaccine WR and vaccine WF for $\mathrm{DPT}+\mathrm{HBV}$ was $17.94 \%$ and $1.22 \%$, respectively. This data again shows a significant decline in vaccine $\mathrm{WR}$ and vaccine WF for DPT, HBV, and pentavalent after implementation of OVP $(P<.001)$. As mentioned earlier the techniques for the administration of DPT, HBV, and pentavalent vaccines are similar and the administrative guidelines have not changed except for introduction of the OVP.

There is a decline in number of injections provided to the children in 2014 as compared to 2012 as majority of the children are now receiving a single injection of pentavalent vaccine, instead of double injections of DPT and HBV vaccine in the earlier vaccination schedule.

Table 2 shows estimated used doses of OPV, DPT, HBV, and pentavalent vaccine based on the WF of year 2012 and 2014 ie, before and after implementation of OVP. This estimation indicates that after implementation of OVP, an estimated 747727 doses of OPV and 343725 doses of DPT, HBV, and pentavalent have been saved in Surat city in the year 2014. It needs mention that the vaccination techniques and administrative guidelines remain the same among all of the UHCs surveyed, except for the introduction of the pentavalent vaccine and the OVP.

\section{Discussion}

According to the WHO, the acceptable vaccine WR for liquid vaccines is $25 \% .{ }^{9}$ The Ministry of Health and Family Welfare, Government of India has also recommended that vaccine WR of all vaccines should not be higher than $25 \%$ (vaccine WF of $1.33 \%) .{ }^{10}$ The vaccine WR for OPV in the present study (after implementation of OVP) is $13.62 \%$ which is lower than the recommended limits, ${ }^{9,10}$ while in the previous study (before implementation of OVP) the vaccine WR was $25 \%$ which was just equal to the recommended wastage limits. ${ }^{8}$

Most freeze-dried (lyophilized vaccines) do not contain preservatives and consequently must not be kept for more than the manufacturers recommended limit and never longer than 6 hours after they are reconstituted. ${ }^{11}$ Liquid injectable vaccines such as DPT, TT, DT, pentavalent, HBV, and liquid formulations of $\mathrm{Hib}$ vaccines contain preservatives that prevent growth of bacterial contamination. ${ }^{11}$ Thus, the OVP is applicable to liquid injectable vaccine and OPV only. The vaccine WR for DPT, HBV, and pentavalent is combinedly $8.05 \%$ in the present study which is much lower than the recommended limits, ${ }^{9,10}$ while in the previous study, ${ }^{8}$ the vaccine WR for DPT and HBV was combinedly $17.94 \%$ which was almost double as compared to the present study. The vaccine WR for all liquid injectable vaccines affected by OVP are also lower in this study as compared with the vaccine WR of other studies. ${ }^{12-14}$

Among all the reasons of vaccine wastage, "residual vaccine left in the vial" was the most frequently reported reason for wastage of vaccines reported by earlier authors. ${ }^{15,16}$ OVP permits the use of open multi-dose vials of vaccines for extended periods of time (up to 28 days), previously which had to be discarded at the end of vaccination session. The use of this residual vaccine is the reason for the improvement in vaccine WR.

According to Mehta et $\mathrm{al}^{8}$ vaccine wastage was also found to be more where sessions are smaller in size. In smaller sessions, more vaccine doses are left over at the end of the sessions which used to be discarded earlier. Now, OVP permits utilization of these left over doses in subsequent sessions which leads to an improvement in vaccine WR and at the same time ensures the vaccine safety.

Based on the economic computation model by Lee et al, ${ }^{2} \mathrm{OVP}$

Table 2. Estimation of Total Saved Doses in Surat City in a Year After Introduction of OVP

\begin{tabular}{|c|c|c|}
\hline Vaccine & OPV & DPT, HBV, and Pentavalent \\
\hline Vaccine WF before implementation of OVPa (See reference 6) & 1.33 & 1.22 \\
\hline Vaccine WF after implementation of OVPb & 1.16 & 1.09 \\
\hline Estimated vaccine doses consumed in SMC during $2014^{c}$ & 6785840 & 3516040 \\
\hline Projected vaccine doses wasted in 2014 if OVP was not implemented ${ }^{d}$ & 1683705 & 634040 \\
\hline Estimated vaccine doses wasted in 2014 after implementation of OVPe & 935978 & 290315 \\
\hline Estimated vaccine doses saved due to implementation of OVP & 747727 & 343725 \\
\hline
\end{tabular}

Abbreviations: OPV, oral polio vaccine; OVP, open vial policy; HBV, hepatitis B virus; SMC, Surat Municipal Corporation; WR, wastage rate; WF, wastage factor. ${ }^{a}$ Vaccine WF according to the study done by Mehta et al ${ }^{8}$ in Surat city during January-March 2012.

${ }^{b}$ Vaccine WF calculated in this study from the data of January-March 2014. Vaccine WF was calculated by using the formula Vaccine WF $=[100 /(100-V a c c i n e$ WR)].

'Estimated vaccine doses consumed was calculated from data provided by immunization cell of SMC which include vaccine provided through 40 urban heath centers (UHCs) across the city. Vaccine consumption of April-September 2014 was doubled to estimate the yearly consumption of vaccine doses for all 40 UHCS of SMC.

${ }^{d}$ Total vaccine doses wasted in absence of OVP was calculated by taking the vaccine WF of year $2012 .{ }^{8}$ Vaccine doses wasted was calculated by the formula 'Total doses wasted' $=$ Total consumption $x[1-(1 /$ vaccine WF $)]$.

e Total vaccine doses wasted after implementation of OVP was calculated by taking the vaccine WF of year 2014. Wasted doses were calculated by formula 'Total doses wasted' $=$ Total consumption $\mathrm{x}[1-(1 /$ vaccine WF $)]$. 
has the potential to reduce vaccine WR up to $30 \%$, without affecting the immunization coverage. In present study, vaccine WR was reduced $45.52 \%$ and $55.13 \%$ for OPV and injectable (DPT, HBV, and pentavalent) vaccines, respectively, and at par with the prediction of the computation model. The above-mentioned modeling also predicts annual saving of $\$ 40$ million worldwide. ${ }^{3}$ In the present study, 747727 doses of OPV and 343725 doses of injectable (DPT, HBV, and pentavalent) vaccine could be saved. Taking approximate cost of OPV ${ }^{11}$ and pentavalent vaccine ${ }^{18}$ INR 3.6 and INR 120.0 per dose, respectively, an estimated annual cost saving is INR 43.94 million ( $\$ 0.70$ million taking conversion rate 62.46 dated March 26th, 2015). For all doses of DPT, HBV, and pentavalent saved, the unit cost of pentavalent has been considered because most of the vaccine doses were of this type.

\section{Conclusion}

Implementation of OVP has significantly reduced vaccine wastage of OPV and liquid injectable vaccines (DPT, HBV, and pentavalent) for Surat city. The WRs of OPV and injectable vaccines reduce by $50 \%$ after introduction of this new policy. The reduction in vaccine wastage by application of the OVP has helped Surat city by an estimated savings of $\$ 0.7$ million in our current vaccination drive. The vaccine WRs as calculated in this study could prove useful for program managers to forecasts their vaccine requirements, provided other factors affecting open vial's usability and administration systems remain unchanged.

\section{Limitations}

In this study, we could not cover all health centers owing to reasons as ascribed. This being a non-systemic exclusion should not affect the results of the study. In the study, all estimations have been based on the information obtained for a period of three months and on this basis yearly forecasts have been made. It needs mention that these three months were common for both the studies for the pre and post OVP. This study covers a large numbers of vaccination sessions so exclusions of the remaining sessions are anticipated to have very little effects on the outcome. Changes in vaccines efficacy after introduction of OVP need to be evaluated separately as this variable has not been included in this study.

\section{Acknowledgements}

Surat Municipal Corporation, Surat, GJ, India Dr. Shreyash Mehta.

\section{Authors' contributions}

Study conception and design: PBP and JJR; Acquisition of data: JJR, SJJ, MJP, and NRB; Analysis and interpretation of data: PBP, JJR, NRB, and SJJ; Drafting of manuscript: PBP, JJR, and MJP; Critical revision: RKB, PBP, and JJR.

\section{Ethical issues}

The study was approved by Institutional Ethical committee (IEC), SMIMER Medical College, Surat, GJ, India.

\section{Competing interests}

Authors declare that they have no competing interests.

\section{References}

1. Mukherjee A, Das V, Shrivastava A, et al. An assessment of wastage multiplier factor and percent wastage of vaccines during routine immunization under UIP, Govt. of India. J Vaccines Vaccin. 2010;4(3):181-185. doi:10.4172/2157-7560.1000181

2. Lee BY, Norman BA, Assi TM, et al. Single vs. multi-dose vaccine vials: an economic computational model. Vaccine. 2010;28(32),5292-5300. doi:10.1016/j.vaccine.2010.05.048

3. World Health Organization (WHO). The use of opened multi-dose vials of vaccine in subsequent immunization sessions. Geneva, Switzerland: Department of Vaccines and Biologicals: 2000;1-8. https://www.spc.int/phs/pphsn/Outbreak/Vaccine_Management/ WHO-Multi_Dose_Vial_Policy.pdf

4. World Health Organization (WHO). Monitoring vaccine wastage at country level: Guidelines for programme managers. Geneva, Switzerland: The Department of Immunization, Vaccines and Biological: 2003. http://www.spc.int/phs/pphsn/Outbreak/ Vaccine_Management/WHO-Monitoring_Vaccine_Wastage_at_ the_Country_Level.pdf

5. Ministry of Health, HSIMT Division, Program Evaluation Unit, Canada. Panorama Vaccine Inventory Module, Vaccine Wastage Report 2013. https://www.infoway-inforoute.ca/en/component/ edocman/1706-panorama-vaccine-inventory-module-vaccinewastage-report-ministry-of-health/view-document?ltemid=188

6. Temptime Corporation, USA. The public health benefit of detecting vaccines exposed to damaging temperatures. http:// www.temptimecorp.com/resources/case-studies/the-publichealth-benefit-of-detecting-vaccines-exposed-to-damagingtemperatures/

7. Ministry of Health and Family Welfare, Immunization Division, Government of India, New Delhi. Open Vial Policy for DPT, TT, HepB, OPV and Liquid Pentavalent. http://www.searo.who.int/ india/topics/routine_immunization/Operational_Guidelines_for_ introduction_Hib_as_Pentavalent_vaccine_2013.pdf. Published 2013

8. Mehta S, Umrigar P, Patel P, Bansal R. Evaluation of vaccine wastage in Surat. National Journal of Community Medicine. 2013;4(1):15-19.

9. World Health Organization (WHO). Immunization service delivery \& accelerated disease control. http://www.who.int/ immunization_delivery/vaccine_management logistics/logistics/ expected wastage/en/index.html. Cited April 20, 2014.

10. Department of Health and Family Welfare, Ministry of Health and Family Welfare, Government of India. Immunization Handbook for Medical Officers. http://nihfw.org/pdf/nchrc-publications/ immunihandbook.pdf. Published 2008.

11. UNICEF. Vaccine Wastage Assessment. Field assessment and observations from National stores and five selected states of India 2010. http://www.unicef.org/india/Vaccine_Wastage_ Asses sment_India.pdf. Cited April 20, 2014.

12. Palanivel C, Kulkarni V, Kalaiselvi S, Baridalyne N. Vaccine wastage assessment in a primary care setting in urban India. Journal of Pediatric Sciences. 2012;4(1):e119.

13. Guichard S, Hymbaugh $\mathrm{K}$, Burkholder $\mathrm{B}$, et al. Vaccine wastage in Bangladesh. Vaccine. 2010;28(3):858-863. doi:10.1016/j. vaccine.2009.08.035

14. Mukherjee A, Ahluwalia T, Gaur L, et al. Assessment of vaccine wastage during a Pulse Polio Immunization Programme in India. J Health Popul Nutr. 2004;22(1):13-18.

15. Linkins $R$, Mansour $E$, Wassif $O$, Hassan $H$, Patriarca $P$. Evaluation of house-to-house versus fixed-site oral poliovirus vaccine delivery strategies in a mass immunization Campaign in Egypt. Bull World Health Organ. 1995;73(5):589-595.

16. World Health Organization (WHO). Open Vials. http://extranet. who.int/ivb_policies/reports/open_vials.pdf. Cited April 20, 2014.

17. World Health Organization (WHO). WHO Policy Statement: Multi-dose Vial Policy (MDVP). Available from: http://www.who. int/immunization/documents/en/. Published in September 2014.

18. Pentavalent boost to immunization. The Times of India. 2014. http://timesofindia.indiatimes.com/city/jaipur/Pentavalent-boostto-immunization/articleshow/38710907.cms. Cited January 12, 2015. 